\title{
Técnica de impresión para rebordes móviles. Parte 1: maxilar
}

\section{Impression Technique of movable fibrous ridge.Part 1:Maxilla.}

\author{
Felipe San Martín-Forray ${ }^{1^{*}}$
}

1. Unidad de Prótesis Removible. Servicio de Sanidad Dental ,Sub dirección Odontológica, Hospital de Carabineros HOSCAR, Chile.

${ }^{*}$ Correspondencia autor: Felipe San Martín Forray. | Antonio Varas 2500, clínica $18,3^{\circ}$ piso .Edificio Corporativo, Nuñoa ,Santiago, Chile. | Fono: 996980277-222441172 | e-mail:ghpaisaje@gmail.com Trabajo recibido el 03/07/2017. Aprobado para su publicación el 27/08/2017

\section{INTRODUCCION}

La retención, el soporte y la estabilidad de una prótesis total dependen de la óptima calidad y fidelidad de la impresión funcional realizada a los tejidos de soporte ${ }^{(1)}$. Esto se dificulta por la presencia de zonas de reborde mucoso móvil (RM) producidas por la reabsorción extrema del reborde óseo, provocando el desalojo de la prótesis. La prevalencia de RM varía entre $5 \%$ en mandíbula y $24 \%$ en el maxilar ${ }^{(1-2)}$. Se caracteriza por un cordón fibromucoso de diámetro variable ubicado generalmente sobre el remanente óseo anterosuperior. Su etiología es diversa y se puede manifestar como secuela post traumática por pérdida masiva de hueso alveolar o reacción a la injuria protésica crónica. Esto último se relaciona con el "Síndrome de Combinación" en el cual una prótesis tota superior ocluye sólo contra el grupo dentario anteroinferior remanente, provocando la hiperplasia de la mucosa y la reabsorción del hueso subyacente antero superior, entre otras características ${ }^{(3)}$.

Las alternativas de manejo de RM comprenden cirugía preprotética de remoción, prótesis implanto asistida y prótesis removible convenciona sin cirugía previa ${ }^{(1)}$.El resultado post quirúrgico no es predecible y puede acarrear la pérdida de superficie útil del reborde y del fondo del vestíbulo, perjudicando la estabilidad de la prótesis ${ }^{(1-2-4)}$. La opción implantosoportada dependerá de las condiciones sistémicas del paciente y está limitada por el escaso hueso remanente ${ }^{(2)}$.

La rehabilitación protésica convencional sin cirugía previa se inicia con una impresión cuyo objetivo es comprimir selectivamente las zonas de soporte maxilar sin desplazar ni distorsionar el área de RM; ya que si es presionado en función tenderá a recuperar su forma y posición desalojando la prótesis ${ }^{(1-2-5)}$.Diversas técnicas de impresión para RM se han propuesto: cubetas individuales abiertas o "ventanas" que dejan a descubierto el RM logrando la impresión en dos tiempos; perforadas o articuladas -conformadas por dos o más partes que se ensamblan al momento de la impresión en boca-. Hacen uso de combinaciones de materiales de impresión: yeso parís, ceras, pasta zinquenólica hidrocoloide irreversible, compuesto de modelar y siliconas de distintas densidades. Algunas son complejas ya que demandan varios pasos o tiempos operatorios; usan materiales de difícil manejo ya que deben se trabajados simultáneamente, no permitiendo controlar la presión ejercida sobre el RM incorporando distorsiones al modelo final de trabajo y finalmente resultando incómodas para paciente y operador ${ }^{(1-6-7-8-9)}$.

Este reporte describe una alternativa nueva y simplificada de impresión para reborde móvil maxilar.

\section{DESCRIPCION DE CASO}

Sujeto de 67 años, ASA 1.Desdentado total superior, parcial inferior con remanencia de dientes anteroinferiores, con indicación de exodoncias por periodontitis avanzada severa y mal pronóstico protésico. Portador de prótesis total superior desajustada durante ocho años. No reporta uso de prótesis inferior. Marcada atrofia ósea alveolar y presencia de reborde móvil antero superior con superficie mucosa sana. Se planifica la confección de prótesis total superior y prótesis total inferior inmediata previa a las exodoncias. Es notificado del tratamiento a realizar, proporciona el consentimiento informado del Hospital de Carabineros de Chile, Santiago y autoriza la toma de fotografías. (Fig.1)

Se realiza impresión preliminar con cubeta de stock e hidrocoloide irreversible (Jeltrate, USA). Con bisturí se espacia el área de RM y se rebasa con hidrocoloide irreversible de consistencia fluida (Fig.2).

Sobre el modelo de estudio, se cubre el área de RM con tres láminas de cera a fin de espaciar y se confecciona cubeta individual acrílica con mango en el centro palatino (Fig.3 a-b). Los bordes se ajustan en boca a la zona marginal neutra vestibular y al límite palatino posterior con fresón de carbide. Mediante compuesto de modelar (Kerr, USA) se efectúa la impresión de la zona de sellado periférico, sin prolongarlo hacia el área de RM. Con el mismo material se impresiona la zona de sellado principal palatino, extendiéndolo hacia el paladar duro (Fig. 3 c).Se comprueba el grado de retención obtenido traccionando la cubeta, la cual debe ejercer resistencia al desalojo. Se perfora la zona anterior y posterior del espaciado con fresa redonda de carbide de 1 milímetro de diámetro y se pincela toda la cubeta con adhesivo para silicona (Coltene, Suiza) (Fig3 d). Se carga la zona posterior y palatina de la cubeta con silicona de polivinilsiloxano de adición (3M, USA) a través de pistola y se asienta y estabiliza en boca mediante el mango central (Fig.4 a). Se introduce la punta aplicadora en los agujeros vestibulares del espaciado 

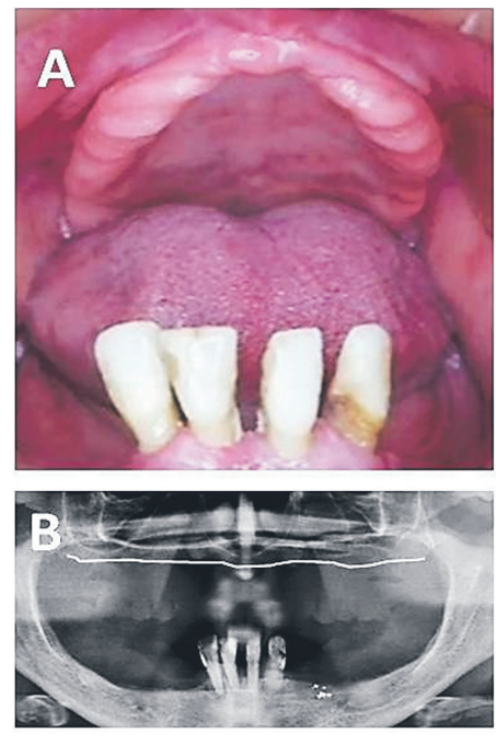

Fig.1. a: Desdentado total superior, con reborde móvil anterior y extrema reabsorción ósea. Remanencia de dientes anteroinferiores con indicación de exodoncia. b: La radiografía muestra el mínimo nivel óseo (línea blanca).Nótese condición de reborde y piezas remanentes mandibulares.

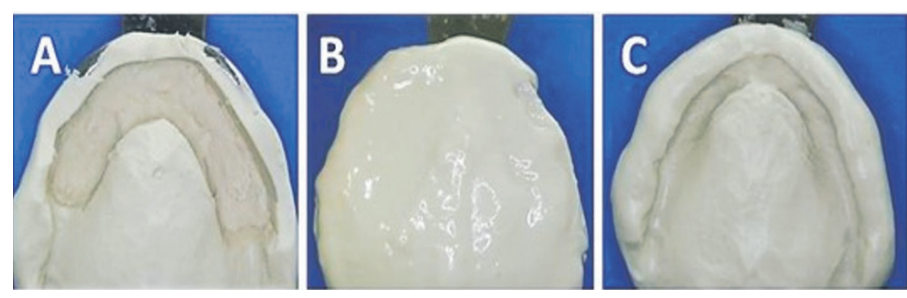

Fig.2. a: Impresión preliminar espaciada en zona de reborde móvil.b: Rebasado con hidrocoloide irreversible fluido. c: Impresión terminada.
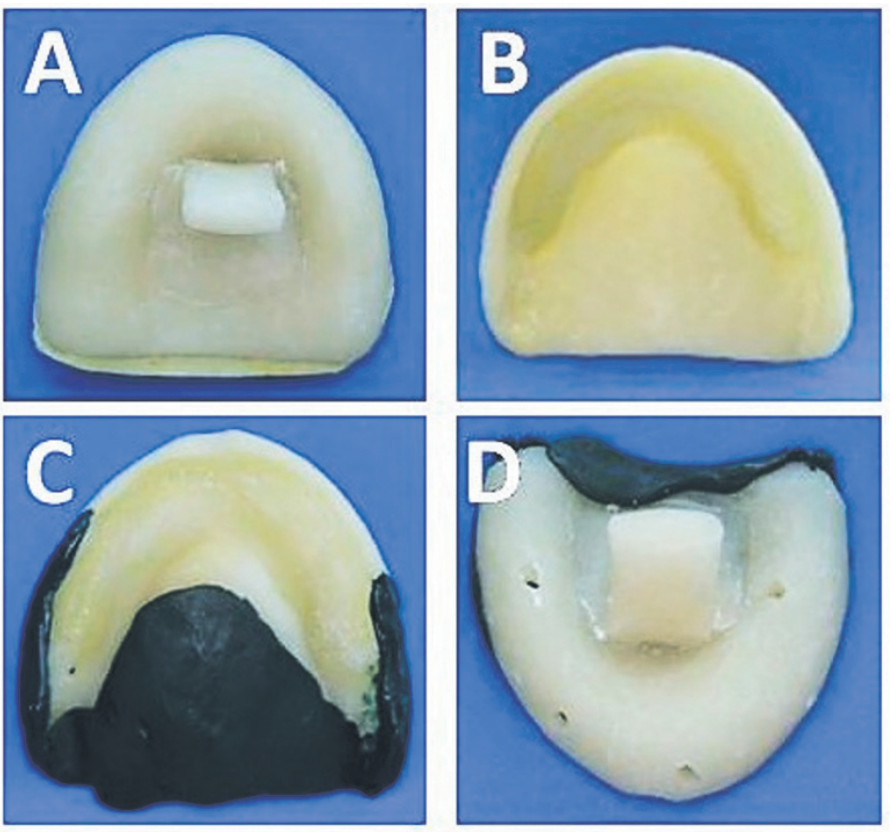

Fig.3. a: Cubeta individual con mango central. b: Espaciado en zona de reborde móvil. c:Impresión de zonas de sellado periférico y principal con compuesto de modelar. Nótese la extensión sobre toda el área palatina. $\mathrm{d}$ :Perforaciones vestibulares y palatinas del espaciado

rellenando con silicona hasta verificar la salida del exceso de material por los agujeros palatinos; se repite la operación a través de estos últimos (Fig. 4 b). Se complementa rellenando por todo el borde vestibular anterior. Durante el tiempo de trabajo del material, se efectúan suaves movimientos rotatorios de labios y mejillas guiados por el operador, a fin de registrar la actividad muscular hasta su polimerización total. Se retira la cubeta de boca, se lava, se critica la impresión, se reposiciona en boca para verificar su retención y estabilidad (Fig.5). Se lava y desinfecta, se encofra y se obtiene el modelo de trabajo sobre el cual se termina la prótesis de manera convencional, dándole un esquema oclusal balanceado bilateral con leve inoclusión del grupo dentario anterior. Se efectúan las exodoncias de los dientes remanentes inferiores, se instalan las prótesis y se realizan controles post-operatorios a fin de verificar el comportamiento de las prótesis, detectar zonas de sobrecompresión y rebasar la prótesis inferior una vez cicatrizados los tejidos (Fig.6).
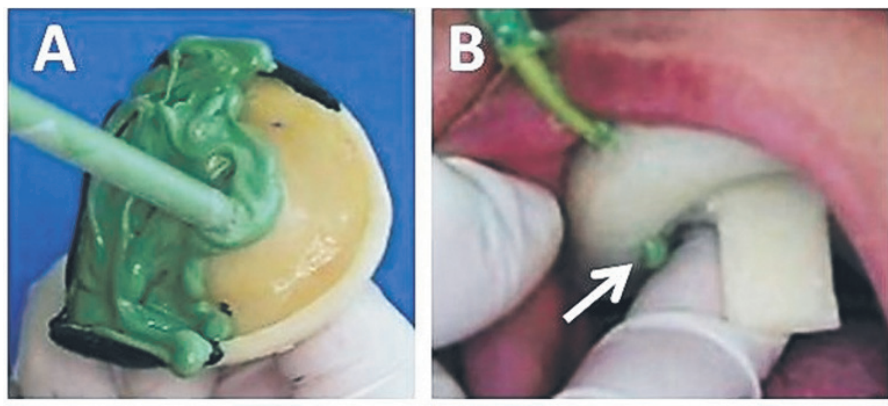

Fig.4. a: Carga de la cubeta con silicona solo en la zona posterior. b:Una vez asentada en boca, relleno del espaciado mediante punta aplicadora. Nótese el escurrimiento de material por el orificio palatino (flecha).

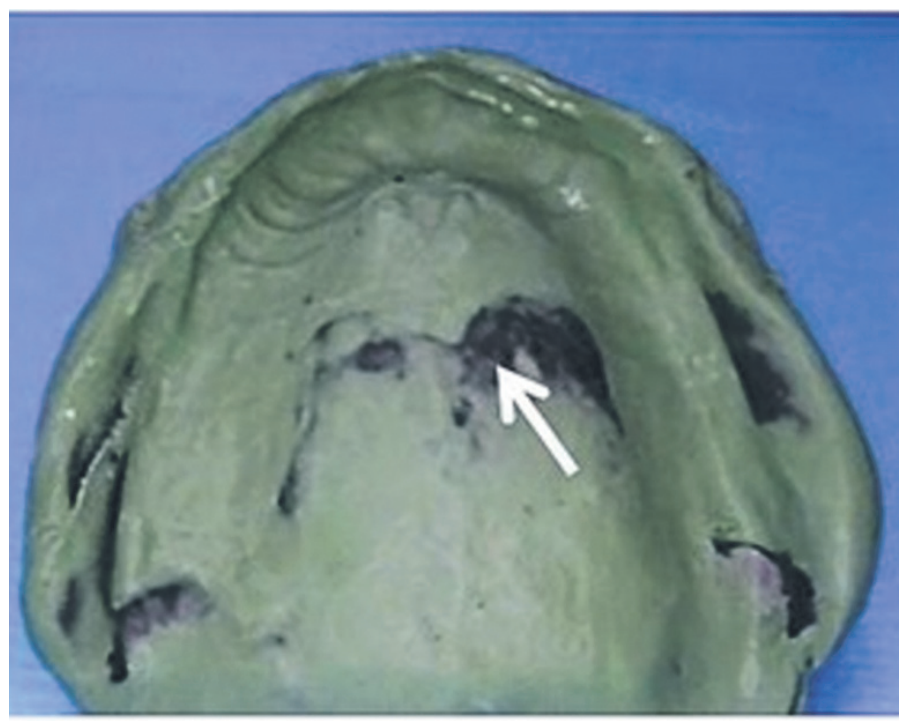

Fig.5. Impresión definitiva. Nótese la continuidad del borde del flanco vestibular y la correcta aplicación de presión de asentamiento, al traslucirse el compuesto de modelar en la zona palatina (flecha).

\section{DISCUSION}

EI RM se deforma bajo presión, por lo que la impresión debe registrarlo de manera estática y transmitir la carga oclusal a la mayor zona de soporte posible ${ }^{(5)}$. En este caso la mucosa se presenta sana, por lo que no es necesario el uso de acondicionador de tejido previo a la impresión preliminar. La consistencia normal del hidrocoloide irreversible deforma el RM, por lo que se espacia la primera impresión y se rebasa luego con una porción de consistencia fluida que no desplaza el tejido. Esta es la impresión preliminar "clásica" para estas situaciones. Kitamura y col. indican que al aumentar la relación agua-polvo en 1,5 veces, la deformación permanente del hidrocoloide disminuye en $2,4 \%$ y la deformación elástica disminuye en $17,6 \%$. Si la relación es 1.75 veces, disminuyen a $1,7 \%$ y $19,1 \%$ respectivamente. Esto se traduce en una impresión preliminar con menor distorsión y mayor fidelidad, obteniéndose a partir de ella un óptimo modelo de estudio sobre el que se confecciona la cubeta individual. ${ }^{(11)}$

El diseño de la cubeta individual acrílica comprende un espaciado sobre RM y adaptación total sobre el resto del reborde y paladar. El mango central posiciona y estabiliza la cubeta, dirigiendo la presión de asentamiento del material de impresión hacia el reborde posterior y toda la superficie palatina, a fin de obtener sellado principal a expensas del 

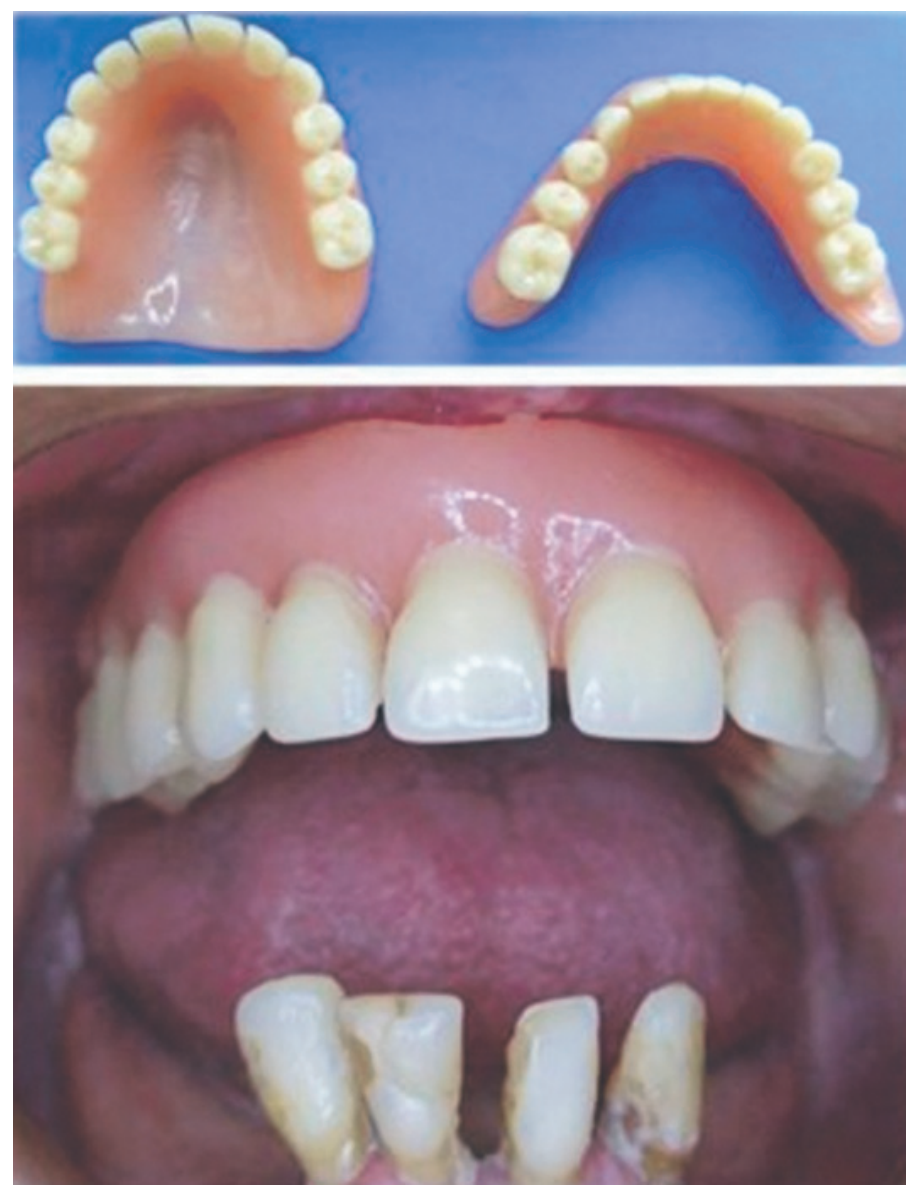

Fig.6. Prótesis terminadas. La prótesis inferior inmediata y se instalará una vez realizadas las exodoncias.

paladar blando y soporte sobre todo el paladar duro (hueso compacto) Es por esto que se extiende la cobertura del compuesto de modelar, modificando la extensión de la zona de soporte, incluyendo el rafe medio (considerado como una zona de alivio); a fin de no transmitir carga al reborde móvil (Fig.5). En caso de producirse úlceras por sobrecompresión estas son aliviadas puntualmente durante los controles post operatorios.

Estos conceptos se basan en los postulados de Krol y Jacobson, que persiguen optimizar la retención, soporte y estabilidad protésica, sustentados en la anatomía del desdentado total y preservando el remanente biológico. ${ }^{(5-14-15)}$

El llenado del espaciado a través de los orificios libera la presión hidráulica de la silicona sobre el RM al escurrir por el agujero contralateral, igualando su magnitud, manteniendo estático el RM. El relleno sobre el borde vestibular anterior y los movimientos funcionales de labio y mejilla, definen el límite del borde protésico anterior. Esta zona no se registra con compuesto de modelar-debido a su alta densidad-, a fin de evitar distorsiones de RM y del fondo de vestíbulo. Así se logra continuidad de todo el flanco y borde protésicos con la zona posterior delimitada con compuesto de modelar (Fig.5).

Se obtiene así una impresión a presión selectiva: mucoestática en la zona del RM y compresiva sobre el reborde posterior y paladar.(12${ }^{13)}$ Sanath y col. indican que si la presión final de asentamiento de una cubeta ajustada es de $100 \%$, la de una cubeta espaciada y perforada es, comparativamente, de sólo un $5 \% .{ }^{(12)}$

La técnica presentada es rápida, simple, limpia, cómoda para el paciente y el operador, y utiliza sólo compuesto de modelar y silicona. Se toma en un solo tiempo, evitándose distorsiones por presión y/o malposición de la cubeta. La silicona no escurre por gravedad, a diferencia de la técnica clásica "de ventana" que comprende tres pasos y más materiales, entre ellos pasta zinquenólica y /o yeso parís de difícil manejo, el cual debe ser sostenido por el operador para que no escurra por la ventana hasta su fraguado; pudiendo producirse distorsiones del material y de la posición tisular ${ }^{(7-9)}$. La modalidad con cubeta multiperforada puede distorsionar el RM ya que la magnitud de presión hidráulica generada es difícil de controlar, pues depende del número, diámetro y ubicación de los orificios; así como de la consistencia del material de impresión ${ }^{(1-12-13)}$. La cubeta articulada comprende dos partes y dos impresiones sucesivas con pasta zinquenólica y silicona, pudiendo producir distorsiones posicionales y demanda un alto nivel de entrenamiento del operador ya que las piezas son ensambladas en boca durante el tiempo de trabajo del material de impresión y del técnico dental -quien confecciona las piezas de la cubeta-(10).

El esquema balanceado bilateral estabiliza la prótesis durante las trayectorias excursivas, evitando la rotación en el plano horizontal. La inoclusión anterior evita la deformación por carga del RM y el "efecto síndrome de combinación", ya que la carga oclusal recae sobre premolares y molares, transmitiéndose al área de soporte palatino y reborde posterior ${ }^{(3-5-54-15)}$.

\section{CONCLUSION}

La técnica de impresión descrita es simple, rápida, utiliza materiales de fácil manipulación y de bajo costo. Controla la presión hidráulica de asentamiento de la cubeta, manteniendo el tejido móvil en posición, evitando su deformación, optimizando la estabilidad de la prótesis completa.

\section{Bibliografía}

1. Pai UY, Reddy VS, Hosi RN. A single step impression technique of flabby ridges using monophase polyvinylsiloxane material:a case report. Case Rep Dent. 2014;2014:104541.

2. Lynch CD, Allen PF. Management of the flabby ridge: using contemporary materials to solve an old problem. Br Dent J. 2006;200(5):258-261.

3 . Kelly E. Changes caused by a mandibular removable partial denture opposing a maxillary complete denture. J Prosthet Dent. 2003;90(1):213-219.

4. Ali M, Chaturvedi S, Shah M. Innovative tunnel technique for mandibular flabby ridge. Int J Dent Clin. 2012; 4(2):74-76.

5. Jacobson TE, Krol AJ. A contemporary review of the factors involved in completes dentures. Part III: support. J Prosthet Dent.1983;43(3):306-13.

6 . Hadjieva H, Dimova M. Selective pressure impressions methods for total dentures by patients with loose and hypermobile mucosa on the alveolar ridges. J IMAB. 2005;11(2):51-53.

7. Sonawane Y, Gangadhar S, Bandhari A, Baldawa S. Grab the flab, window impression technique: a case report. IOSR JDMS. 2013;11(4):39-42.

8. Agrawal B, Agrawal S, Mangal A. Management of flabby ridge: A case report IJDS. 2011;(2,3):20-23.
9. Rashid H, Naz A, Ahmed A, Zuhaib M. Revisiting the window technique. JPDA. 2015;24(4):1-10.

10. Crawford R, Walmsley A. A review of prosthodontic management of fibrous ridges. Br Dent J. 2005; 199(11):715-719.

11. Kitamura $A$, Kawai $Y$. Basic investigation of the laminated alginate impression technique: setting time, permanent deformation, elastic deformation, consistency and tensile bond strength tests. J Prosth Res. 2015;59:49-54.

12. Sanath S, Ratna V, Shemoy K. The selective pressure maxillary impression. A review of techniques and presentation of an alternate custom tray design. JIPS. 2007; 7(1):8-11.

13. Shum M, Pow E. Management of excessive movable tissue: a modified impression technique. J Prosth Dent. 2014; 112: 387-89.

14. Jacobson TE, Krol AJ. A contemporary review of the factors involved in completes dentures. Part II: stability. J Prosthet Dent.1983; 49(2):165-72.

15. Jacobson TE, Krol AJ. A contemporary review of the factors involved in completes dentures. Part I: retention. J Prosthet Dent.1983; 49(1):5-15. 